\title{
Effects of Exogenous Melatonin on Photosynthesis of Chinese Cabbage Seedlings under $\mathrm{NaCl}$ Stress
}

\author{
Yi Tang ${ }^{1, a}$, Renheng Lin ${ }^{1, b}$, Jingyu Lan ${ }^{2, c}$ and Huanxiu Li ${ }^{1, d^{*}}$ \\ ${ }^{1}$ Institute of Pomology and Olericulture, Sichuan Agricultural University, Chengdu, Sichuan, China \\ ${ }^{2}$ College of Horticulture, Sichuan Agricultural University, Chengdu, Sichuan, China \\ atangyisunguochao@sina.com, b412116458@qq.com, '598938381@qq.com, dhxli62@163.com \\ ${ }^{*}$ Corresponding author
}

Keywords: Melatonin; $\mathrm{NaCl}$ stress; Chinese cabbage; Photosynthesis

Abstract. A pot experiment was conducted to study the effects of spraying melatonin (MT) on photosynthesis of Chinese cabbage seedlings under $\mathrm{NaCl}$ stress. The result showed that net photosynthetic rate $(\mathrm{Pn})$, stomatal conductance (Gs), water use efficiency (WUE), light use efficiency (LUE) and transpiration rate (Tr) were improved, which resulted to the photosynthetic capacity enhanced significantly when spraying low concentration of MT. Therefore, exogenous MT could efficiently increase resistance of Chinese cabbage seedlings to $\mathrm{NaCl}$ stress, and the MT concentration of $100 \mu \mathrm{mol} \cdot \mathrm{L}^{-1}$ was the best.

\section{Introduction}

Land salinization is one of serious environmental and socio-economic problems around the world. And it is one of the main restricting industrial developments of China's vegetables [1]. Regulation by exogenous substances can effectively alleviate salt stress damage to the plant [2]. Some studies indicate that exogenous administration of 5-aminolevulinic acid (ALA) [3], salicylic acid (SA) [4] etc. have a regulatory role to plants under $\mathrm{NaCl}$ stress.

Chinese cabbage (Brassica rapa ssp. pekinensis) belong to Brassica vegetable crops. Because of its rich nutrients, loved by the people, and it is the largest cultivated area of a vegetable in China [5]. Melatonin (MT) is indole derivatives of tryptophan [6]. Numerous studies have found that melatonin as an endogenous free radical scavenger showed strong antioxidation effects, can effectively alleviate abiotic stress harm on plant growth and development, such as the heavy metal stress [7], high temperature stress [8], chilling stress [9], UV radiation [10] etc. .

In recent years, research on melatonin has become one of the hot spots at home and abroad. In this study, we used different concentrations of melatonin to spray Chinese cabbage seedlings, for the purpose of exploring the role of exogenous MT in induction of Photosynthetic Characteristics in Chinese cabbage seedlings under $\mathrm{NaCl}$ stress, in the hope of providing references for future research and cabbage cultivation in the salinized land.

\section{Materials and Methods}

Materials. The experiment wasconducted at Sichuan Agricultural University $\left(30^{\circ} 42^{\prime} \mathrm{N}, 103^{\circ} 51^{\prime}\right.$ E), Wenjiang, China. The seeds of Chinese cabbage named quick 35 were harvested in 2014 and purchased from Chengdu, China. All chemicals used in experiments were of analytical grade. Melatonin was purchased from Sigma-Aldrich (St. Louis, MO, USA).

Experimental Design. Seeds were sterilized in 10\% sodium phosphate solution for 30 minutes, flushed five times in distilled water, and then placed on 9-cm-diameter Petri dishes with three layers of filter paper moistened with distilled water and germinated at $25^{\circ} \mathrm{C}$ in darkness. Seeds were considered germinated when the seed coat was broken and a radicle was visible. After germination, seeds were planted in nutrition pot filled with vermiculite and perlite, the pot was ten centimeters in diameter and height.

Seedlings were irrigated with $20 \mathrm{ml}$ Hoagland nutrient solution containing $50 \mu \mathrm{mol} \cdot \mathrm{L}^{-1}$ concentra -tions of $\mathrm{NaCl}$ every other day, until the experiment finished. 
When the third leaf expanded, their leaves were sprayed with 0 (control), 50, 100, 200, 400 $\mu \mathrm{mol} \cdot \mathrm{L}^{-1}$ concentrations of melatonin solution until foliage and dorsal dripping. Seedlings were sprayed with melatonin solution every other day, and three times in total. Each treatment consisted of 10 pots with one plant per pot. Positions of the pots were randomly changed daily to minimize positional effects. 30 days after treatment, the photosynthesis of each plant was determined by using LI-6400 portable photosynthesis meter (LI-COR Inc., USA). The photosynthetic parameters of the photosynthesis meter were manual control $\mathrm{CO}_{2}$ concentration $400 \mu \mathrm{mol} \cdot \mathrm{CO}_{2} \mathrm{~mol}^{-1}$, temperature $25^{\circ} \mathrm{C}$, light intensity $1000 \mu \mathrm{mol} \mathrm{m}{ }^{-2} \cdot \mathrm{s}^{-1}$. The determination of photosynthetic parameters were net photosynthetic rate $(\mathrm{Pn})$, transpiration rate $(\mathrm{Tr})$, stomatal conductance $(\mathrm{Gs})$ and $\mathrm{CO}_{2}$ concentration of intercellular $(\mathrm{Ci})$, and each treatment was repeated three times. Water use efficiency $(\mathrm{WUE})=$ net photosynthetic rate $(\mathrm{Pn}) /$ transpiration rate $(\mathrm{Tr})$, Light use efficiency (LUE) $=$ net photosynthetic rate (Pn) / light intensity[11].

Statistic analyses. Statistical analyses were performed using SPSS 13.0 statistical software (IBM, Chicago, IL, USA). Data were analyzed by one-way ANOVA with least significant difference (LSD) at a $5 \%$ confidence level.

\section{Results and Discussion}

Net Photosynthetic Rate (Pn). Spraying low concentrations of MT could significantly improve the photosynthetic rate of Chinese cabbage seedlings under $\mathrm{NaCl}$ stress (Fig. 1). And with increasing the concentration of MT, the Pn of Chinese cabbage increased first and then decreased. When the concentration of MT was $100 \mu \mathrm{mol} \cdot \mathrm{L}^{-1}$, Pn of Chinese cabbage reached a peak, enhanced Pn by $54.65 \% \quad(p<0.05)$ compared with control. However, there was no significant difference in photosynthetic rate of spraying $200,400 \mu \mathrm{mol} \cdot \mathrm{L}^{-1} \mathrm{MT}$ and control.

Transpiration Rate (Tr). Compared with control, spraying 50 and $100 \mu \mathrm{mol} \cdot \mathrm{L}^{-1} \mathrm{MT}$ were significantly increased $\mathrm{Tr}$ of Chinese cabbage seedlings under $\mathrm{NaCl}$ stress, enhanced $\mathrm{Tr}$ by $26.60 \%$ $(p<0.05)$ and $9.95 \%(p<0.05)$, respectively (Fig. 2). When the MT concentration exceeded 100 $\mu \mathrm{mol} \cdot \mathrm{L}^{-1}, \operatorname{Tr}$ decreased rapidly. When spraying concentration of $400 \mu \mathrm{mol} \cdot \mathrm{L}^{-1}$, the Chinese cabbage seedling transpiration rate lower than control, but no significant difference with control.

Water Use Efficiency (WUE). As shown in Fig. 3, The WUE of Chinese cabbage was increased with the increasing of MT concentration from $0 \mu \mathrm{mol} \cdot \mathrm{L}^{-1}$ to $100 \mu \mathrm{mol} \cdot \mathrm{L}^{-1}$. When the concentration of MT was $100 \mu \mathrm{mol} \cdot \mathrm{L}^{-1}$, the WUE of Chinese cabbage seedlings was the highest. After spraying MT was 200 and $400 \mu \mathrm{mol} \cdot \mathrm{L}^{-1}$, the WUE decreased $5.13 \%(p<0.05)$ and $10.49 \%(p<0.05)$ respectively compared with control.

Light Use Efficiency (LUE). Low concentration OF MT could promote LUE of Chinese cabbage seedlings under $\mathrm{NaCl}$ stress, while excessive $\mathrm{MT}$ would lead to inhibition. The same as Pn, spraying concentration of MT was $100 \mu \mathrm{mol} \cdot \mathrm{L}^{-1}$, the treatment enhanced LUE by $54.65 \%(p<0.05)$, compared with control.

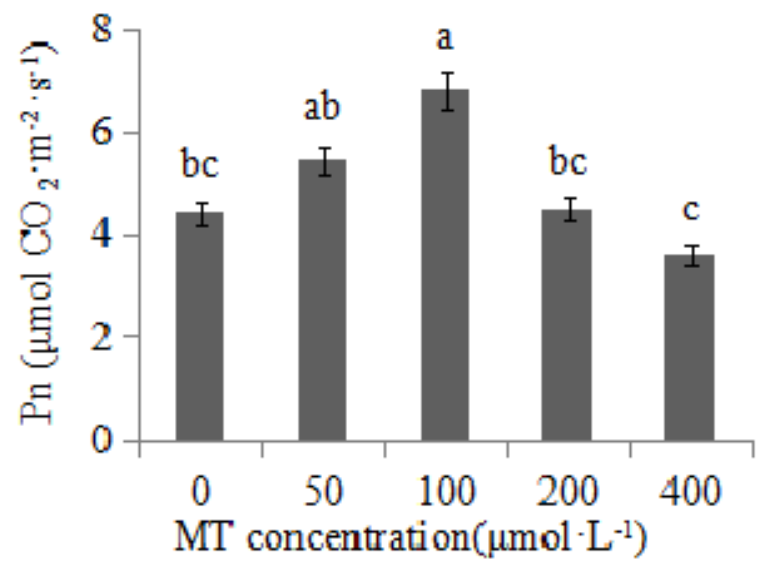

Fig. 1 Pn of MT sprayed Chinese cabbage

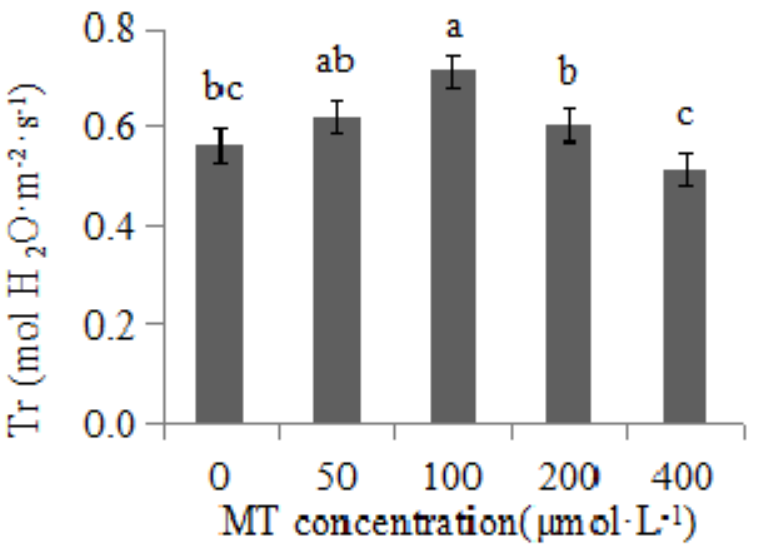

Fig. 2 Tr of MT sprayed Chinese cabbage 


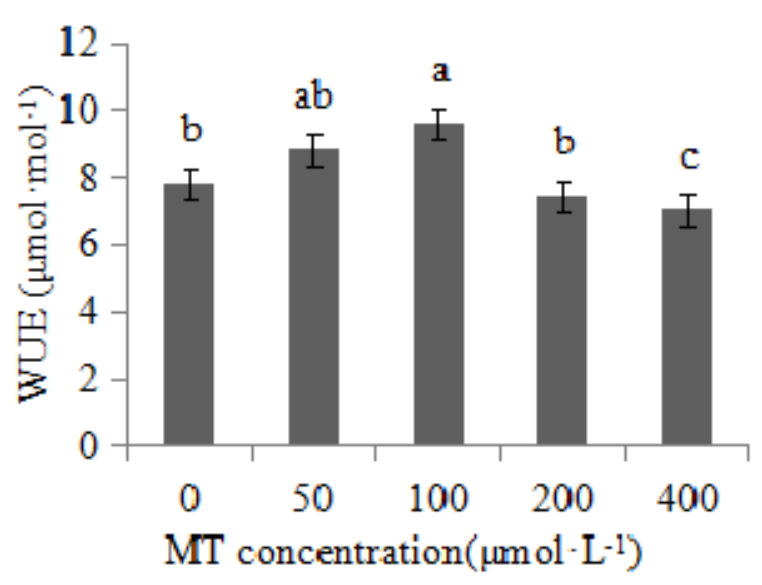

Fig. 3 WUE of MT sprayed Chinese cabbage

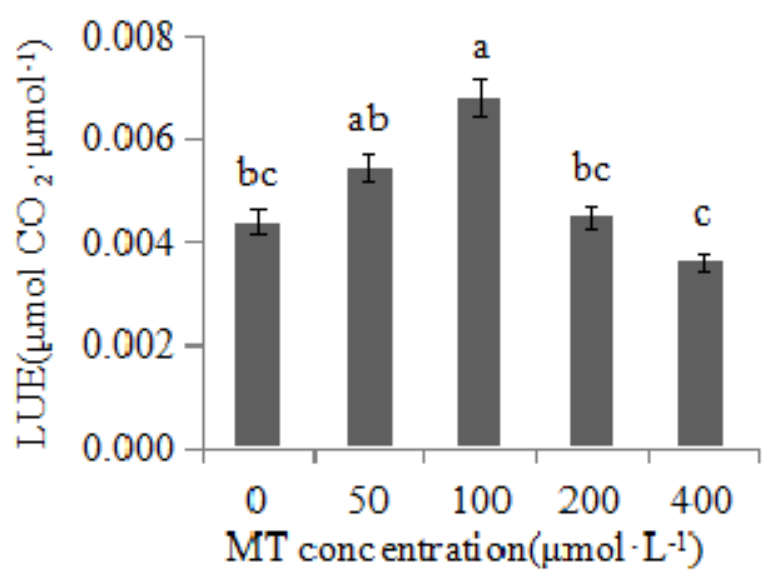

Fig. 4 LUE of MT sprayed Chinese cabbage

Stomatal Conductance (Gs). When the concentration of MT was $100 \mu \mathrm{mol} \cdot \mathrm{L}^{-1}$, the Gs reached a maximum, higher than control, significantly. Then, as the concentration of MT increased, there was a significant reduction of Gs.

$\mathrm{CO}_{2}$ Concentration of Intercellular (Ci). Compared with control, after spraying MT, the Ci of Chinese cabbage increased. But, there were no significant difference with the control.

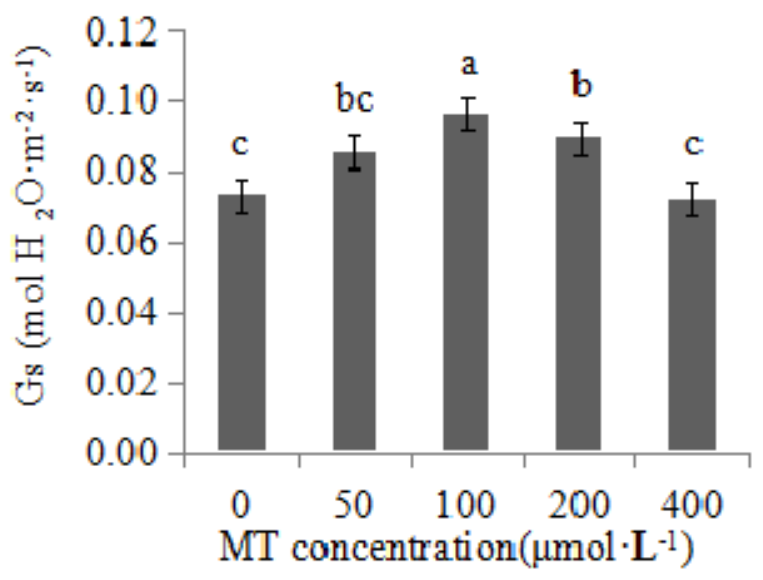

Fig. 5 Gs of MT sprayed Chinese cabbage

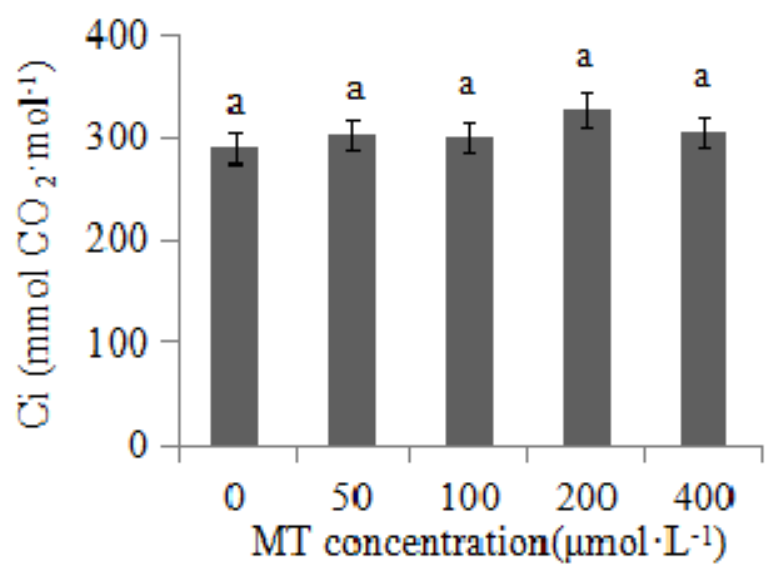

Fig. $6 \mathrm{Ci}$ of MT sprayed Chinese cabbage

\section{Conclusions}

Plant photosynthetic reaction system was damaged under $\mathrm{NaCl}$ stress, which resulted in the inhibition of photosynthesis [12]. The study found that the Pn, Tr, WUE, LUE and Gs of Chinese cabbage seedlings were promoted by low concentration of MT and inhibited by high concentration, the reason of which was a high concentration of MT have toxic effects on the photosynthetic apparatus. To sum up, spraying low concentrations of MT can improve photosynthesis of Chinese cabbage seedlings under $\mathrm{NaCl}$ stress, in addition, the best concentration of MT was $100 \mu \mathrm{mol} \cdot \mathrm{L}^{-1}$.

\section{Acknowledgements}

This work was financially supported by the Sichuan Agricultural University "Shuang-Zhi Plan" Foundation, Sichuan Provincial Department of Education Foundation (15ZA0011).

\section{References}

[1] Y. G. Wang, Y. Li and D. N. Xiao: Environmental Geology Vol.56 (2008), p. 439-446. 
[2] J. Y. Zhu, G. Y. Yang and F. J. Zhao: Journal of Northeast Normal University Vol.35 (2003), p. 105-108 (In Chinese).

[3] Y. Zhou, L. Xu and L. Yang: Journal of Southwest China Normal University Vol.37 (2012), p. 111-115 (In Chinese).

[4] J. L. Li, Q. Liu and S. Li: Journal of South China Agricultural University Vol.31 (2010), p. 42-46 (In Chinese).

[5] F. L. Zhang and J. W. Jian: China Vegetables Vol.3 (2011), p. 1-2 (In Chinese).

[6] A. B. Lerner, J. D. Case and Y. Takahashi: Journal of the American Chemical Society Vol.80 (1958), p. 2587-2587.

[7] M. Posmyk Małgorzata, H. Kuran and K. Marciniak: Journal of Pineal Research Vol.45 (2008), p. 24-31.

[8] T. Iskender, K. Huseyin: Journal of Pineal Research Vol.52 (2012), p. 332-339.

[9] V. S. Bajwa, M. R. Shukla and S. M. Sherif: Journal of pineal research, Vol.56 (2014), p. 238-245.

[10]L. J. Zhang, J. F. Jia and K. Mei: Journal of Nuclear Agricultural Sciences, Vol.5 (2015), p. 830-835 (In Chinese).

[11]X.J. Jiang, H. Wang and W. Peng: Shanxi Journal of Agricultural Sciences Vol. 54 (2008), p.56-58 (In Chinese).

[12]Q. Z. Xing, S. L. Yu and Y. P. Niu: Agricultural Research in the Arid Areas Vol. 29 (2011), p.96-100 (In Chinese). 